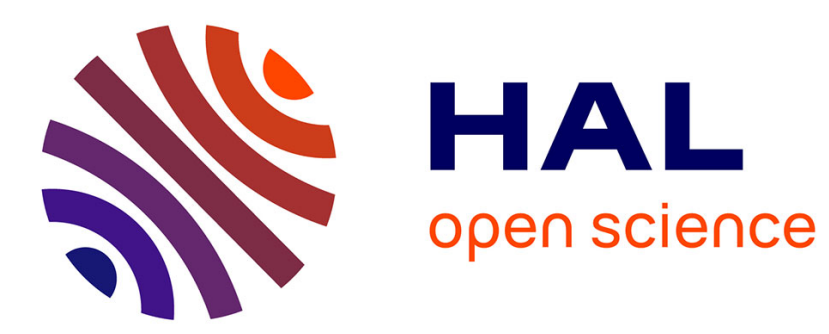

\title{
Nouvelle étude du Lokeśvara khmer du Musée national de Colombo (Sri Lanka)
}

Brice Vincent

\section{To cite this version:}

Brice Vincent. Nouvelle étude du Lokeśvara khmer du Musée national de Colombo (Sri Lanka). Arts Asiatiques, 2013, 68 (1), pp.27 - 38. 10.3406/arasi.2013.1810 . halshs-02477458

\section{HAL Id: halshs-02477458 \\ https://shs.hal.science/halshs-02477458}

Submitted on 13 Feb 2020

HAL is a multi-disciplinary open access archive for the deposit and dissemination of scientific research documents, whether they are published or not. The documents may come from teaching and research institutions in France or abroad, or from public or private research centers.
L'archive ouverte pluridisciplinaire HAL, est destinée au dépôt et à la diffusion de documents scientifiques de niveau recherche, publiés ou non, émanant des établissements d'enseignement et de recherche français ou étrangers, des laboratoires publics ou privés. 
Nouvelle étude du Lokeśvara khmer du Musée national de Colombo (Sri Lanka)

Brice Vincent

\section{Citer ce document / Cite this document :}

Vincent Brice. Nouvelle étude du Lokeśvara khmer du Musée national de Colombo (Sri Lanka). In: Arts asiatiques, tome 68, 2013. pp. 27-38;

doi : https://doi.org/10.3406/arasi.2013.1810

https://www.persee.fr/doc/arasi_0004-3958_2013_num_68_1_1810

Fichier pdf généré le 08/11/2019 


\title{
Résumé
}

Découvert et publié au début des années 1920 par Victor Goloubew, le Lokeśvara khmer du Musée national de Colombo reste un témoignage historique majeur des relations anciennement tissées entre le Cambodge et le Sri Lanka. C'est à ce titre qu'il nous paraît utile de réexaminer cette statuette en bronze : en prenant d'abord en compte des considérations d'ordre technique, iconographique et stylistique, puis en discutant en détail les conditions de sa découverte. Une telle approche aide notamment à mieux comprendre l'hypothèse de V. Goloubew, qui proposa d'identifier l'idole comme un ex-voto offert par le souverain khmer, et Roi lépreux " historique ", Jayavarman VII. À la légende est toutefois préféré le contexte des interactions politiques et religieuses entretenues entre les royaumes khmer et singhalais à partir du milieu du XIle siècle. Le long voyage de l'idole est ainsi attribué à un pèlerin bouddhiste, sans doute venu du Cambodge pour vénérer Avalokiteśvara en un sanctuaire local réputé et dédié au bodhisattva guérisseur.

\begin{abstract}
Discovered and published in the early 1920s by Victor Goloubew, the Khmer Lokeśvara in the Colombo National Museum remains an important historical record of ancient relations between Cambodia and Sri Lanka. It is for this reason that it seems worthwhile to study this bronze statuette again : first taking into account technical, iconographic and stylistic considerations, and then discussing in detail the conditions of its discovery. This particularly helps in understanding $\mathrm{V}$. Goloubew, who proposed identifying the idol as an ex-voto offered by the Khmer ruler, and the historical Leper King, Jayavarman VII. However, the context of political and religious interactions between Khmer and Sinhalese kingdoms from the mid-twelfth century is preferred to legend. The long journey of the idol is thus attributed to a Buddhist pilgrim, who probably came from Cambodia to worship Avalokiteśvara at a renowned local sanctuary dedicated to the healer Bodhisattva.
\end{abstract}




\section{Nouvelle étude du Lokeśvara khmer du Musée national de Colombo (Sri Lanka) ${ }^{1}$}

\section{Résumé}

Découvert et publié au début des années 1920 par Victor Goloubew, le Lokeśvara khmer du Musée national de Colombo reste un témoignage historique majeur des relations anciennement tissées entre le Cambodge et le Sri Lanka. C'est à ce titre qu'il nous paraît utile de réexaminer cette statuette en bronze : en prenant d'abord en compte des considérations d'ordre technique, iconographique et stylistique, puis en discutant en détail les conditions de sa découverte. Une telle approche aide notamment à mieux comprendre l'hypothèse de V. Goloubew, qui proposa d'identifier l'idole comme un ex-voto offert par le souverain khmer, et Roi lépreux « historique», Jayavarman VII. À la légende est toutefois préféré le contexte des interactions politiques et religieuses entretenues entre les royaumes khmer et singhalais à partir du milieu du XII ${ }^{\mathrm{e}}$ siècle. Le long voyage de l'idole est ainsi attribué à un pèlerin bouddhiste, sans doute venu du Cambodge pour vénérer Avalokiteśvara en un sanctuaire local réputé et dédié au bodhisattva guérisseur.

\section{概要}

科倫坡國立博物館收藏的高棉世自在神像是維克多 格羅布在二十世紀二十年 代發現和公佈的, 迄今仍是有關古代柬埔寨和斯里蘭卡之關係的重要歷史見證。 正是由於這個原因, 這尊青銅小雕像似乎值得再做研究, 首先對技術、圖像和風 格進行分析, 然後詳細探討發現銅像的情況。這樣做特別有助於理解格羅布的看 法, 他主張將雕像鑑定為高棉統治者、史載罹患麻風病的國王阇耶跋摩七世貢獻 的還願物。不過, 與其相信傳說, 不如把它放在十二世紀中葉以來高棉和錫蘭王 國之間在政治和宗教上的交流這個背景之中來理解。因而本文將雕像的長途旅 行歸諸一位佛教朝聖者, 毫無疑問他來自柬埔寨, 此行的目的是朝拜當地一座供 奉治病菩薩觀世音的著名聖殿。

\footnotetext{
* Nous tenons d'abord à remercier Osmund Bopearachchi pour les nombreux conseils et facilités qu'il nous a offerts lors de notre séjour d'étude au Sri Lanka en juillet 2008. Nos remerciements vont également aux directeurs de plusieurs institutions de recherche ainsi qu'à leur personnel, qui nous ont accordé toute leur attentions de recherche ainsi qu'à leur personnel, qui nous ont accordé toute leur atten-
tion, tout en répondant toujours au mieux à nos demandes : Nanda Wickremasinghe du Musée national de Colombo, Hab Touch du Musée national du Cambodge à Phnom Penh, Cristina Cramerotti et Isabelle Poujol de la bibliothèque et de la photothèque de l'EFEO, ainsi que R. S. Sundarapandian de la Government Oriental Manuscripts Library de l'université de Madras. Nous remercions enfin tous ceux qui nous ont guidé et conseillé tant dans nos recherches que dans la rédaction de cet article: Gabrielle Abbe, Michel Antelme, David Bourgarit, Hema et Susantha Goonatilake, Michel Jacq-
}

\begin{abstract}
Discovered and published in the early 1920s by Victor Goloubew, the Khmer Lokeśvara in the Colombo National Museum remains an important historical record of ancient relations between Cambodia and Sri Lanka. It is for this reason that it seems worthwhile to study this bronze statuette again: first taking into account technical, iconographic and stylistic considerations, and then discussing in detail the conditions of its discovery. This particularly helps in understanding V. Goloubew, who proposed identifying the idol as an ex-voto offered by the Khmer ruler, and the historical Leper King, Jayavarman VII. However, the context of political and religious interactions between Khmer and Sinhalese kingdoms from the mid-twelfth century is preferred to legend. The long journey of the idol is thus attributed to a Buddhist pilgrim, who probably came from Cambodia to worship Avalokiteśvara at a renowned local sanctuary dedicated to the healer Bodhisattva.
\end{abstract}

\section{要約}

1920 年代初頭にヴィクトール・ゴルベフによって発見、公開された、コロンボの 国立博物館蔵のクメールのローケシュヴァラ像は、古い時代におけるカンボジア とスリランカの間の関係を証明する重要な歷史的遺物である。それ的え、この小 像はあらためて研究される価值があるものと思われる。われわれは、まず技術的、 図像学的、様式的考察を行ない、その後、それが発見された状況について詳しく検 討する。それによって、なぜゴロベフが、これをクメール王朝の有名な「癩王」ジヤ ヤヴァルマン 7 世が、願の成就のために奉納した像だと考えたか、といらことが理 解できる。とは言っても、われわれとしては、この伝説よりも、12 世紀以降のクメ 一ルの各王国とシン八ラとの宗教的、政治的関係の文脈を重視すべきであること を主張する。この像がたどった長い旅は、おそらくカンボジアから出発して病気 を治寸神として広く信仰された、その土地の観音菩薩に参詣したある仏教徒の巡 礼によるものだと思われるのである。

Hergoualc'h, Claude Jacques, Anusha Kasthuri, Madabushi Sampath Kumar, Daniel Negers, Natalie Ong, Christophe Pottier, Peter Sharrock, Dominique Soutif et Arjuna Thantilage.

1. Cet article est issu d'une communication présentée en septembre 2008 à Leyde, aux Pays-Bas, dans le cadre de la $12^{\text {th }}$ International Conference of the European Association of Southeast Asian Archaeologists (EurASEAA 12), et intitulée: « New study of a Khmer bronze statuette of Lokeśvara in the Colombo National Museum, Sri Lanka ». 
C'est en visitant le Musée national de Colombo en janvier 1922 que Victor Goloubew, historien de l'art et membre de l'École française d'Extrême-Orient (EFEO), identifie parmi les bronzes qui y sont conservés une statuette khmère représentant le bodhisattva Lokeśvara ${ }^{2}$. Après l'avoir à nouveau examinée en février 1923, il publie l'année suivante dans le Bulletin de l'École française d'Extrême-Orient (BEFEO) une courte notice de trois pages, où il donne de la sculpture une première étude iconographique et stylistique, tout en fournissant diverses informations relatives aux conditions de sa découverte ${ }^{3}$. Un simple dessin au trait complète cette description et constitue pendant longtemps le seul document disponible pour son étude ${ }^{4}$.

Intéressant à divers titres les recherches de V. Goloubew, la statuette est encore citée dans plusieurs articles de l'auteur, avant de disparaître des publications scientifiques ${ }^{5}$. Du côté des études srilankaises, elle ne semble guère non plus avoir suscité l'intérêt des chercheurs, car aucune mention n'en est faite tant dans les travaux relatifs aux collections du musée de Colombo que dans les grandes synthèses consacrées à l'art bouddhique du Sri Lanka.

Elle n'en reste pas moins un témoignage historique essentiel pour comprendre les relations anciennement tissées entre le Cambodge et le Sri Lanka. C'est à ce titre qu'il nous a paru important de réétudier et de documenter de façon aussi exhaustive que possible cette idole khmère de Lokeśvara, très largement tombée dans l'oubli depuis les travaux pionniers de V. Goloubew.

\section{Technique}

La statuette du musée de Colombo représente une divinité masculine à quatre bras (caturbhuja), avec tous ses attributs encore en place $^{6}$ (fig. 1a-c) ${ }^{7}$. Elle est figurée debout, en position frontale avec le corps légèrement désaxé vers la droite, et repose sur une petite base carrée. Avec ce socle qui en est solidaire, elle mesure environ $24 \mathrm{~cm}$ de haut: il s'agit donc d'un objet essentiellement mobile.

\footnotetext{
2. Selon le Main Register du musée de Colombo, la statuette est entrée dans ses collections le 13 décembre 1915 (15.105.314). Elle était toujours conservée dans ses réserves lors de notre dernière visite au musée en juillet 2008.

3. Goloubew 1924. L'auteur mentionne aussi la statuette un an plus tôt dans un rapport adressé à Louis Finot, alors directeur de l'EFEO (BEFEO, 23, 1923, p. 560).

4. Ce dessin a été réalisé d'après une photographie du musée de Colombo, qui est aujourd'hui conservée à la photothèque de l'EFEO à Paris (fonds Cambodge, réf. CAM 15926).

5. Finot et Goloubew 1930, p. 629, 642 : Goloubew 1930, p. 462-463 : Goloubew 1935, p. 566. Les rares mentions dont fait l'objet la statuette par la suite ne font que reprendre les travaux de V. Goloubew : BEFEO, 25 (1-2), 1925, p. 277 ; BRIGGS 1951, p. 232; JACQUES 1967, p. 122 ; CHANDLER 1979, p. 59; BAPTISTE et ZÉPHIR 2008, p. 250 ; ZÉPHIR 2012, p. 408, n. 15.

6. L'état de conservation décrit a été observé lors de notre dernier passage à Colombo en 2008, avant que la pièce ne soit restaurée par le laboratoire du musée.

7. Les photographies sont dues à l'auteur sauf mention contraire.
}

La statuette a été réalisée selon le procédé de la fonte à la cire perdue, une technique connue et maîtrisée des bronziers khmers depuis au moins l'époque préangkorienne. Elle a été fondue en creux, très certainement à l'envers comme l'indiquent les amas de métal visibles sous le socle, aux quatre angles et au centre. En l'absence d'analyse radiographique cependant, il reste difficile de savoir si certaines parties spécifiques, comme la tête, les bras et les pieds, sont solides, ou encore si les bronziers ont eu recours à des armatures pour façonner le noyau ${ }^{8}$. Des aspérités de couleur rouille, observables en surface dans le milieu du dos et sur le pan arrière du vêtement, font en tout cas penser à des traces d'armature en fer. Il faut en outre remarquer la présence d'un très net défaut de coulée au dos de la statuette, matérialisé par un trou béant, ainsi qu'un autre manque de fonte sur le dessus du socle, au niveau de l'angle postérieur gauche. Ce dernier a été rebouché lors du travail de reparure à l'aide d'une petite plaquette métallique quadrangulaire, aujourd'hui disparue.

La surface de la statuette est en grande partie recouverte de traces vertes de corrosion du cuivre, alors que des dépôts de salissures de couleur blanche occupent les principales jonctions et rainures. Le métal montre également en plusieurs endroits (visage, torse, dos, vêtement et jambes) une teinte nettement brune avec des reflets brillants, comme s'il avait été poli. Peutêtre est-ce là le résultat des multiples usages dont a fait l'objet la statuette, ou, plus vraisemblablement, d'un nettoyage mécanique consécutif à sa découverte, ce dont pourraient également témoigner diverses traces d'outil. Des vestiges très réduits de dorure restent malgré tout visibles, suggérant que la surface a été entièrement dorée, sans doute dès l'origine.

Ajoutons encore qu'un caractère unique, sans doute la consonne $k a$ du vieux khmer, a été inscrit sur le côté gauche du socle, exactement en son centre (fig. 2). Les traits qui le composent semblent avoir été gravés en plusieurs fois, sur le métal et non sur le modèle en cire. Si des marques inscrites reproduisant un caractère plus ou moins similaire s'observent sur quelques images en pierre (fig. 3a-b), aucune autre n'est attestée pour la statuaire en bronze. Des chiffres inscrits sont en revanche bien connus, avec plusieurs fonctions possibles. Tout d'abord, pour les statues et objets en bronze constitués d'éléments coulés séparément, ils ont clairement un rôle de marques d'assemblage, destinées à faciliter le remontage après la fonte ${ }^{9}$. D'autres chiffres inscrits ne seraient toutefois que symboliques, en particulier

8. Si aucune radiographie n'est disponible, la statuette a en revanche fait l'objet d'un prélèvement de métal, réalisé sous le socle, sans doute pour analyse élémentaire. Ce prélèvement s'inscrirait dans le cadre d'une campagne d'échantillonnage menée dans les années 1970 par l'université de Pennsylvanie sur la collection de bronzes du musée de Colombo. Malheureusement, les résultats des analyses ne semblent jamais avoir été publiés (communication personnelle d'Arjuna Thantilage, docteur en archéométallurgie à l'université de Kelaniya de Colombo, juillet 2008).

9. VincENT 2012, p. 260-264. Comme nous le verrons plus loin (note 26), il n'est pas impossible que la marque inscrite étudiée ait été destinée à l'ajustement de la statuette sur un piédestal. 

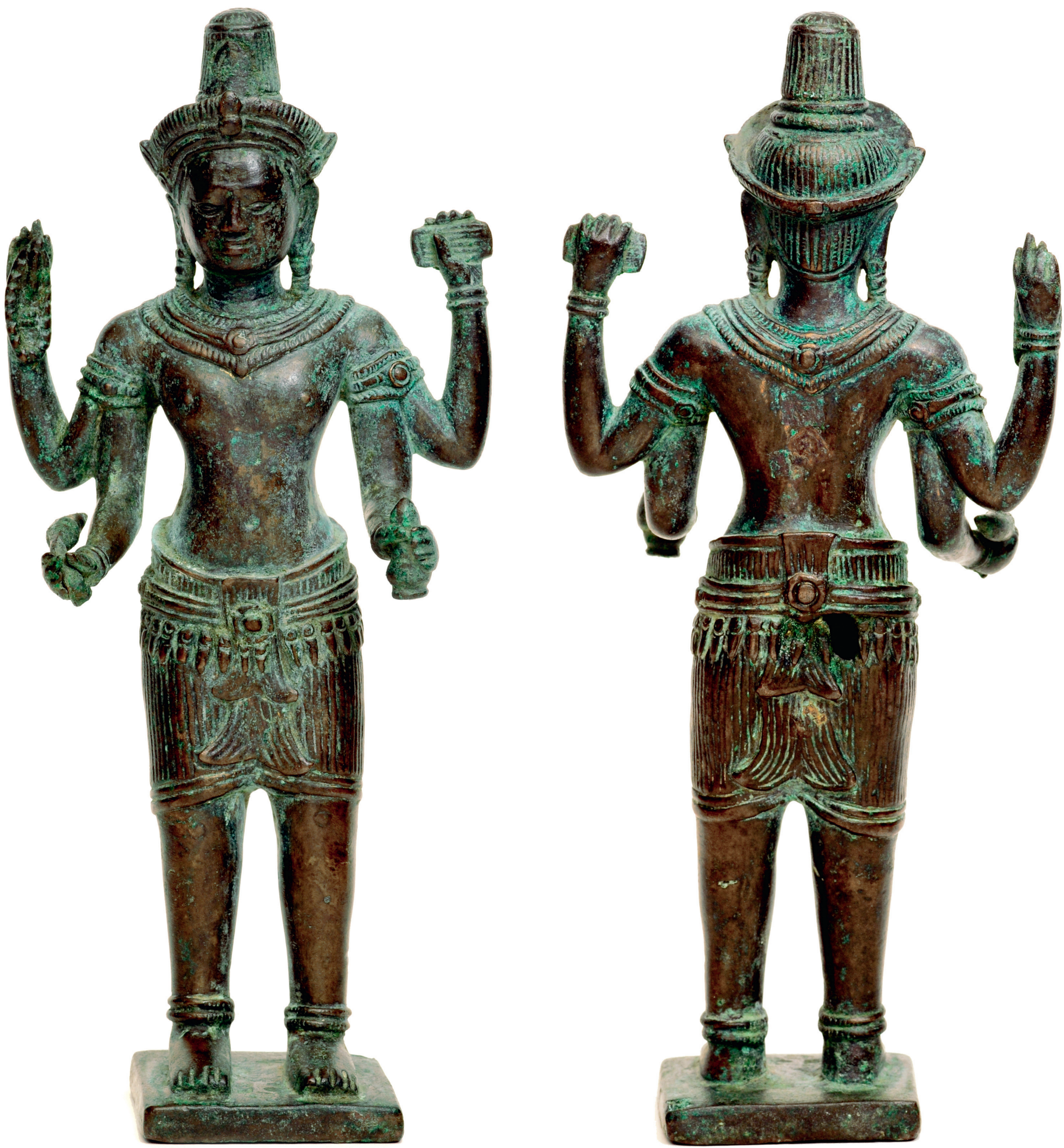

Figures 1a et 1b. - Lokeśvara (face et dos), prolongement du style d’Angkor Vat, fin XII ${ }^{\mathrm{e}}$-début XıII ${ }^{\mathrm{e}}$ siècle, Kōvilavatta, Dondra, district de Matara (Sri Lanka). Bronze avec traces de dorure, H. 23,5 cm; L. 10,5 cm; épaisseur 6,5 cm; poids 1100 g. Musée national de Colombo, 15.105.314. Photos Musée national de Colombo. 


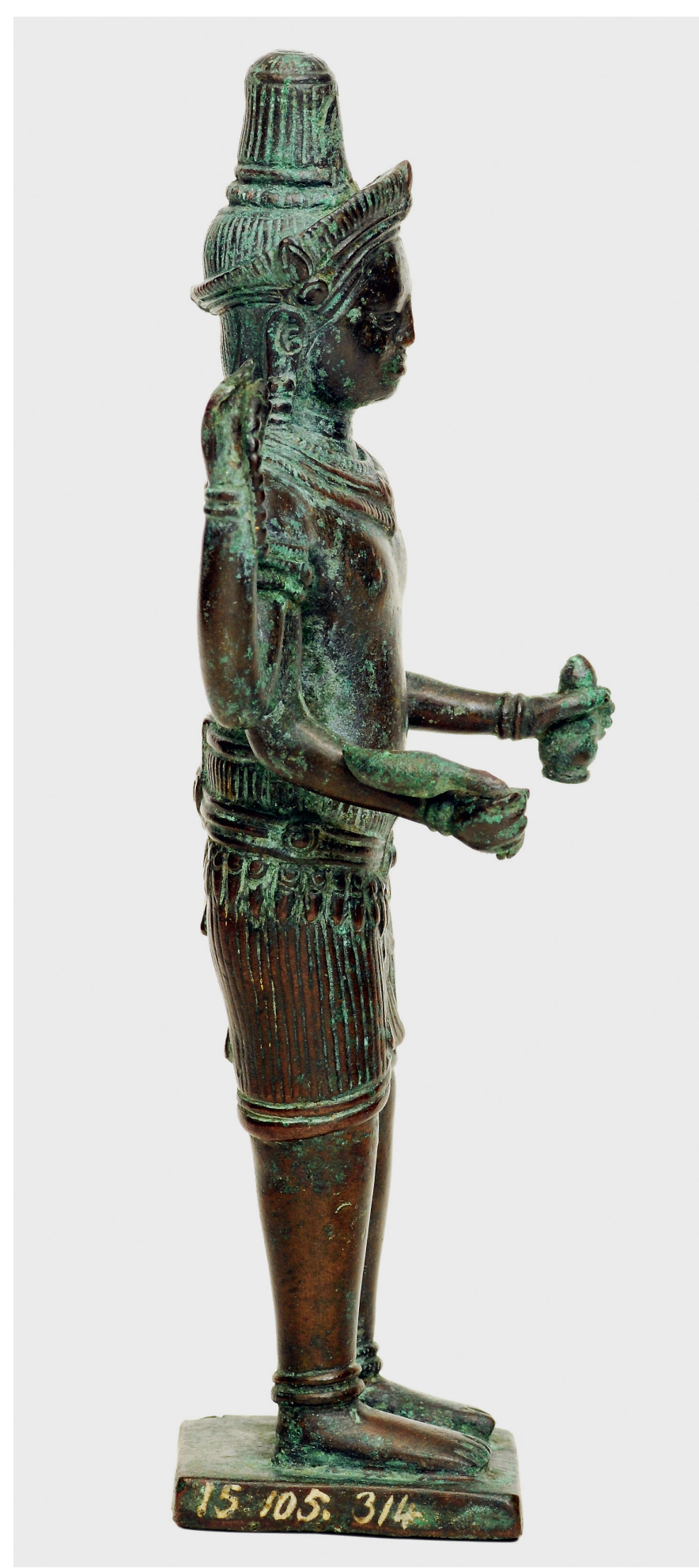

Figure 1c. - Lokeśvara du Musée national de Colombo (cf. fig. 1a-b), côté droit. Photo Musée national de Colombo. dans le cas des images bouddhiques ${ }^{10}$. À moins encore qu'à l'instar des marques de potiers observées sur certaines céramiques khmères, ils n'indiquent un lieu de production, ou même l'atelier où ces statues et objets inscrits ont été fondus ${ }^{11}$. Chacune de ces hypothèses peut s'appliquer au cas étudié.

\section{Iconographie}

V. Goloubew est le premier à reconnaître dans la statuette du musée de Colombo une représentation typiquement khmère du bodhisattva Lokeśvara ${ }^{12}$. Jusqu'à sa visite, elle était identifiée à tort comme une forme de Brahmā, sans doute par confusion avec d'autres représentations de ce dieu connues au Sri Lanka, sous la forme de petites images elles aussi en bronze et à quatre bras ${ }^{13}$.

L'auteur fonde son identification sur les attributs que porte la divinité, tous caractéristiques du bodhisattva Lokeśvara : dans les mains inférieures, la fleur de lotus à droite (padma) - le seul attribut mobile - et le vase à eau à gauche (kamandalu); dans les mains supérieures, le rosaire à droite (akșamālā) et le livre à gauche $(\text { pustaka })^{14}$. Un élément essentiel de son iconographie est toutefois absent: le Buddha Amitābha, habituellement figuré à l'avant de sa coiffure. V. Goloubew a sans doute raison de ne pas y prêter attention, son identification étant confortée par la présence d'un aplat en forme d'ogive sur le devant du chignon cylindrique (jațā), qui ne peut correspondre qu'à une ancienne image miniature du jina Amitābha ${ }^{15}$ (fig. 4). De la même manière qu'un Lokeśvara du Musée national du Cambodge, lui aussi en

10. Cette idée a notamment été avancée par Hiram Woodward à propos d'un ensemble de bronzes bouddhiques khmers, quatre Buddha et un Lokeśvara, eux aussi inscrits au niveau de leur socle (WooDWARD 1980, p. 159-162, 171-172, fig. 10-18 [erratum in WOODWARD 1981, p. 64, n. 71]; cf. aussi VINCENT 2012, cat. 92-95).

11. Il s'agit là d'une hypothèse formulée par Bernard Philippe Groslier dans son article fondateur pour l'étude des céramiques khmères (GrosLier 1995, p. 23). Selon lui, les marques inscrites avant l'enfournement sur le fond plat des céramiques auraient aidé à comptabiliser après cuisson les céramiques produites par un même village ou par un même atelier de potiers, avant que celles-ci ne soient expédiées à leurs destinataires. L'épigraphie khmère donnerait, en effet, l'image d'un mode de production artisanal où les villages étaient «spécialisés dans telle ou telle fabrication d'objet, et devaient en remettre périodiquement des quantités fixées aux autorités, pour stockage puis distribution ».

12. Lokeśvara, le "Seigneur du monde ", est l'épithète qui, à partir du vü ${ }^{\mathrm{e}}$ siècle, est la plus en faveur pour désigner le bodhisattva dans l'épigraphie khmère (CHUTIWONGS 2002, p. 218).

13. GoloubEw 1924, p. 510 («Prajāpati-Brahmā»). Cette première identification comme un Brahmā était toujours adoptée lors de notre dernière visite au musée. 14. Goloubew 1924, p. 511-512.

15. Si cet élément n'est pas utilisé par V. Goloubew dans sa notice de 1924, il signale néanmoins l'existence d'un autre Lokeśvara en bronze sans Buddha Amitābha dans sa coiffure, renvoyant à une des planches des Bronzes khmèrs de George Cœè̀s (1923, p. 48, pl. XXV-2). Comme G. Cœdès le rappelle lui-même, son identification n'est toutefois pas assurée, l'image en question se confondant davantage avec une représentation du dieu Vișnu. À notre connaissance, les seules statues censées figurer le bodhisattva Lokeśvara sans pour autant porter le jina Amitābha sur le devant de leur chignon, sont deux bronzes conservés au musée Guimet (MA 5960 et MA 509; BAPTISTE et ZÉPHIR 2008, p. 249-251 [cat. 73], 444 [cat. 266]). Le premier pose cependant problème, en raison de ses bras fondus séparément qui semblent constituer des ajouts modernes ou, en tout cas, plus tardifs (VINCENT 2012, p. 334 [cat. 122]). 


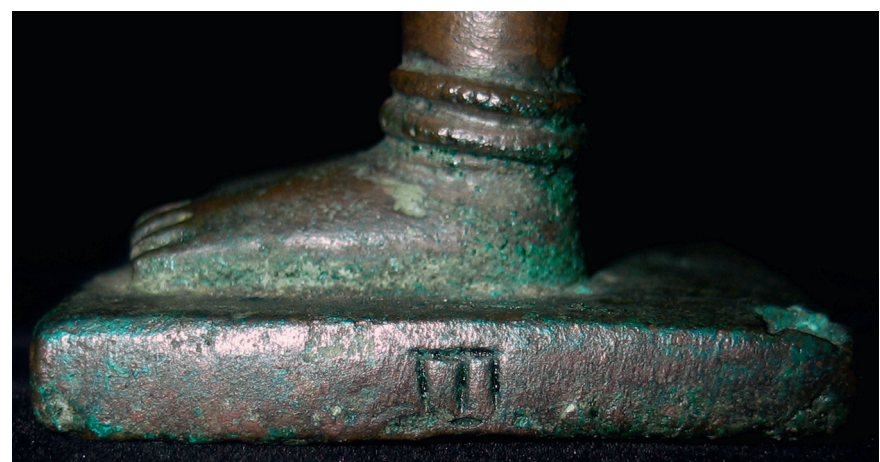

Figure 2. - Lokeśvara du Musée national de Colombo (cf. fig. 1a-b), détail de la marque inscrite gravée sur le côté gauche du socle ( $k a$ ?)

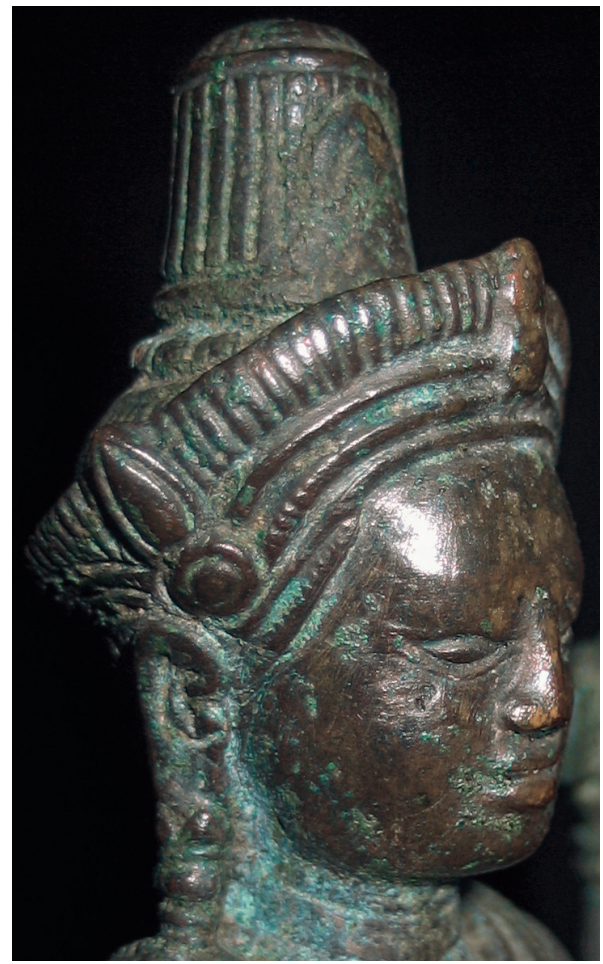

Figure 4. - Lokeśvara du Musée national de Colombo (cf. fig. 1a-b), détail du visage et de la coiffure.

$$
\begin{array}{r} 
\\
\text { Figure 3a. - Triade } \\
\text { bouddhique } \\
\text { figurant Buddha et } \\
\text { la Prajñāpāramitā } \\
\text { (incomplète), style } \\
\text { d'Angkor Vat, } \\
\text { troisième quart du } \\
\text { XII }{ }^{\text {s }} \text { siècle, Vat Damrei } \\
\text { Sar, Battambang } \\
\text { (Cambodge). Grès, } \\
\text { H. } 40 \text { cm. Musée } \\
\text { provincial de } \\
\text { Battambang, ka 134. } \\
\text { Figure } 3 b .- \text { Triade } \\
\text { bouddhique } \\
\text { du Musée provincial } \\
\text { de Battambang } \\
\text { (cf. fig. 3a), détail de } \\
\text { la marque inscrite } \\
\text { gravée à l'arrière } \\
\text { du socle (ka?). }
\end{array}
$$

bronze mais transformé en Śiva ${ }^{16}$, la statuette aurait ainsi été reprise, à une époque qui reste encore à définir. Dans le cas qui nous occupe cependant, seul le Buddha Amitābha aurait été supprimé, et non les attributs comme pour ce qui est du Lokeśvara du musée de Phnom Penh.

Toujours est-il que la statuette du musée de Colombo reprend à l'origine le type iconographique le plus souvent adopté à l'époque angkorienne pour représenter le bodhisattva Lokeśvara - avec quatre bras et les attributs précédemment décrits -, et attesté aussi bien dans l'épigraphie que dans la statuaire et le décor architectural ${ }^{17}$.

16. Ga $5340 ; c f$. VINCENT 2012, cat. 62.

17. Chutiwongs 2002, p. 235.
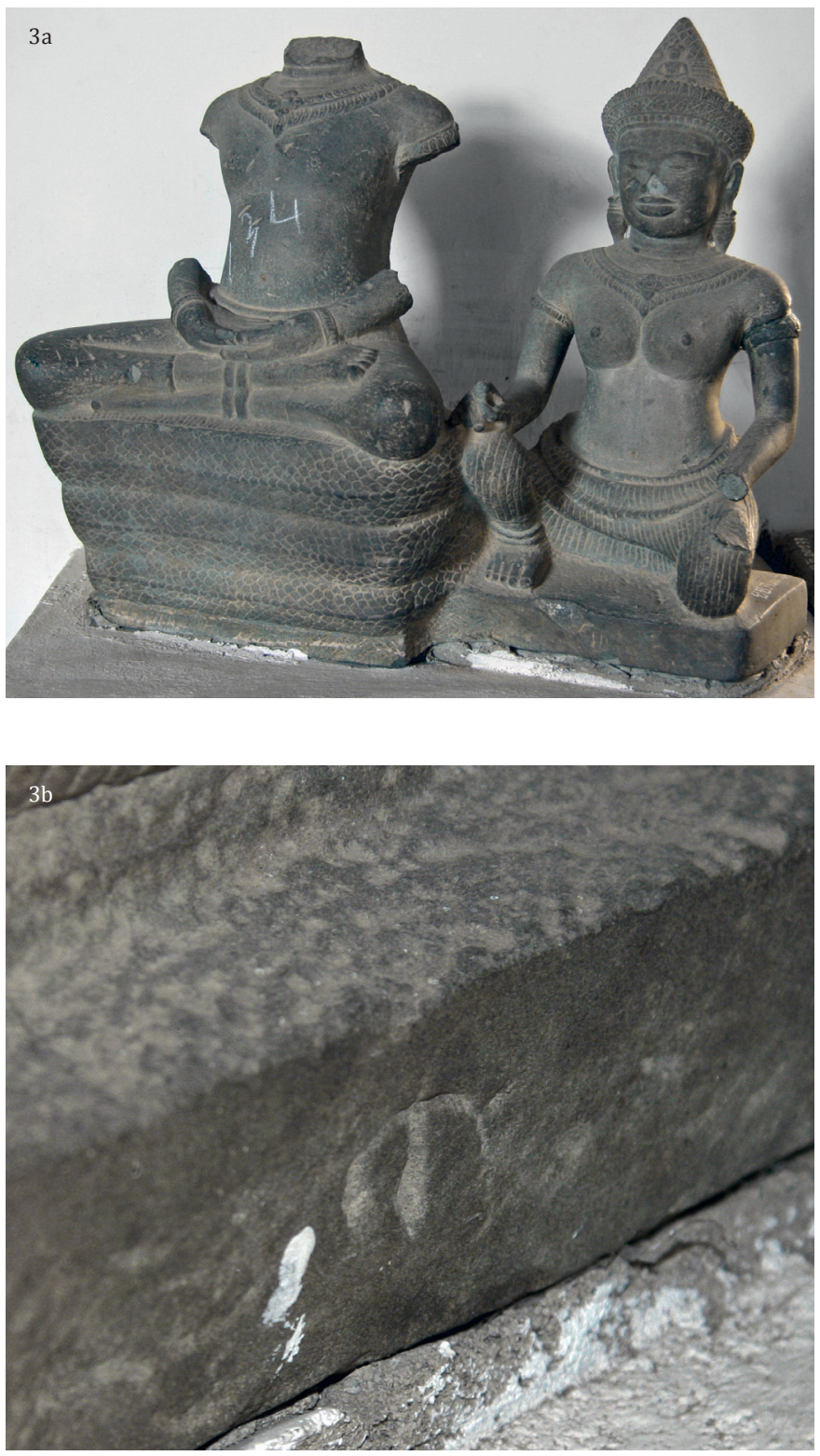

Style

V. Goloubew attribue d'abord cette statuette à un art khmer dit « classique », la datant du IX ${ }^{\mathrm{e}}$ ou du X $\mathrm{X}^{\mathrm{e}}$ siècle, selon une chronologie alors communément admise ${ }^{18}$. Publiées en 1927, les recherches de Philippe Stern sur l'évolution de l'art khmer l'obligent toutefois à revoir cette datation et, dans un article légèrement postérieur, il la rattache finalement $\mathrm{au} \mathrm{XI}^{\mathrm{e}} \mathrm{ou} \mathrm{au}$ $\mathrm{XII}^{\mathrm{e}}$ siècle ${ }^{19}$. Si les arguments qu'il avance dès sa première étude restent valides, il semble possible d'affiner son analyse, en prenant en compte les nombreux progrès accomplis depuis plusieurs décennies dans la connaissance de la statuaire khmère.

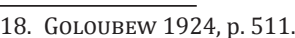

19. GoloubEw 1930, p. 462 
Il est d'abord permis d'inscrire la statuette du musée de Colombo dans la tradition du style d'Angkor Vat (ca.1080-1175). En témoignent sa frontalité et ses proportions générales, avec de larges épaules et un traitement relativement maladroit des membres inférieurs : les jambes sont raides, les pieds informes et les rotules indiquées de manière fantaisiste. Cette tradition stylistique transparaît également à travers le modelé du visage, dont la face est presque inscrite dans un carré, ainsi que dans la richesse de la parure composée d'un diadème, de pendants d'oreilles, d'un collier, de brassards et bracelets, d'une ceinture à pendeloques et d'anneaux de chevilles. Plusieurs éléments, tels le diadème à fleurons devenu couronne ou encore le sampot à double « chute en ancre » devant et derrière, inviteraient toutefois à rattacher cette statuette davantage à la fin du style d'Angkor Vat, et donc à la dater du troisième quart du XII ${ }^{\mathrm{e}}$ siècle.

Il est malgré tout possible qu'elle ait été produite quelques décennies plus tard, sous le règne du souverain bouddhiste Jayavarman VII (r. 1182/1183-ca. 1220), alors même qu'apparaît une nouvelle tradition stylistique dite du Bayon (ca. 1180$1220)^{20}$. Comme le rappelle en effet Albert Le Bonheur à propos des bronzes des styles d'Angkor Vat et du Bayon, il convient toujours de «dissocier style et chronologie, le style pouvant être continué ou repris très postérieurement à sa phase d'épanouissement $»^{21}$.

On connaît ainsi au moins un exemple d'une statuette de Lokeśvara en bronze, en tous points comparable à celle du musée de Colombo et qui s'inscrit donc comme elle dans la tradition stylistique d'Angkor Vat, que l'on peut dater de l'époque du Bayon. Cette statuette fait partie d'une triade bouddhique inscrite, conservée dans une collection privée de Singapour, où le bodhisattva est figuré à la droite du Buddha, alors que la Prajñāpāramitā est à sa gauche (fig. 5). Il s'agit là du type iconographique le plus en faveur au cours du règne de Jayavarman VII, que ce soit dans la statuaire ou le décor architectural ${ }^{22}$. Outre l'iconographie, une courte inscription en vieux khmer gravée sur la face antérieure du socle commun de la triade, au-dessous du Buddha central, permet de confirmer la date de sa production.

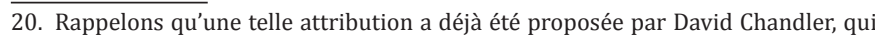
concède également que la statuette du musée de Colombo reste assez difficile à dater (CHANDleR 1979, p. 59).

21. Le BONHEUR 1972, p. 129, n. 4.

22. Chutiwongs 2002, p. 229-230.
Elle mentionne en effet l'installation d'une divinité nommée Śrī Jaya, qui, d'après l'étude que fait George Cœdès d'une inscription identique, elle aussi gravée sur une triade mahāyānique en bronze (K. 945), serait à identifier comme « Jayavarman VII dont la plupart des fondations portent justement un nom débutant par celui du roi $»^{23}$.

Certes, à l'encontre d'une telle attribution à l'époque du Bayon, il pourrait être objecté que, contrairement au Lokeśvara de la triade de Singapour, celui de Colombo ne faisait pas partie à l'origine d'une triade, ce qu'indique l'absence de tenon sous son socle ou de tout autre moyen de fixation. Ce serait toutefois oublier que le bodhisattva Lokeśvara fait également l'objet au cours du règne de Jayavarman VII de représentations indépendantes, non seulement en pierre mais aussi en bronze. En témoigne notamment une statuette mise au jour à Angkor dans la région de Roluos, au lieu-dit de Veal Kralanh, au sein d'une cache contenant quatre autres bronzes du style du Bayon $^{24}$ (fig. 6a-b). Aujourd'hui conservée au Musée national du Palais de Taipei, sous la forme d'une idole montée sur un petit socle carré, elle reposait originellement sur un piédestal à cuve à ablutions de plus grandes dimensions ${ }^{25}$. L'ensemble n'était donc consacré qu'au seul culte du bodhisattva Lokeśvara, une orientation religieuse dont la statuette du musée de Colombo, au style très semblable, était sans doute également porteuse, qu'elle ait été ou non posée sur un piédestal du même type ${ }^{26}$.

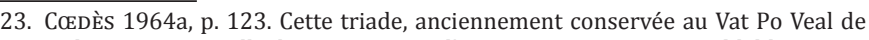
Battambang, comme celle de Singapour et d'autres en tous points semblables issues de diverses collections publiques et privées, feront prochainement l'objet d'une publication (VINCENT, «La triade inscrite du Brooklyn Museum of Art», à paraître). 24. ZÉPHIR 2012, p. 408-409, fig. 5.

25. Il ne fait en effet aucun doute que le Lokeśvara de Veal Kralanh, connu par des archives photographiques du musée Guimet (fonds Bernard Philippe Groslier), mais aussi de la photothèque de l'EFEO (fonds Cambodge, réf. CAM 3251_1 [fig. 6a], 3251_2 [fig. 6b], 18776_1, 18776_2), corresponde à une statuette en bronze khmère publiée dans un catalogue du Musée national du Palais (Ho et al. 2004, p. 63, 320 [cat. 36]). Acquis à une date indéterminée par le collectionneur taïwanais M. Peng Kai-dong avant d'être offert au musée en 2004, il a donc nécessairement quitté le territoire cambodgien plusieurs mois ou années après sa découverte, qui a eu lieu en 1962 ou 1964. Peut-être a-t-il disparu lors du transfert en 1970 de tous les bronzes de la Conservation d'Angkor vers le Musée national du Cambodge à Phnom Penh. Toujours est-il que son piédestal à cuve à ablutions est encore conservé au sein de cette dernière institution ( $g a$ 2516) de même que trois bronzes issus de la cache de Veal Kralanh (VINCENT 2012, cat. 51 [ga 2424], 52 [ga 2494], 76 [ga 5630]), alors que le quatrième a été plus récemment transféré au Musée national d’Angkor à Siem Reap (VINCENT 2012, cat. 64 [ga 2514]).

26. À ce propos, il est intéressant de rappeler la présence d'une marque inscrite sur le socle de la statuette. En tant que marque d'assemblage, elle aurait pu en effet servir à réunir l'idole à son piédestal après la fonte séparée de chacun de ces éléments - à condition que le piédestal soit lui aussi inscrit -, ou encore à centrer cette même image sur la cuve à ablutions. 


\section{Conditions de découverte}

Selon les Ceylon Administration Reports pour l'année 1915 - déjà cités par V. Goloubew -, la statuette provient de l'extrême sud du Sri Lanka et, plus précisément, du village de Dondra, ou Devundara en singhalais, dans le district de Matara (fig. 7). En l'état actuel des recherches, il s'agit du seul objet de facture khmère mis au jour sur l'île.

La même source précise que sa découverte s'est faite de manière fortuite au cours de travaux de défrichage, en un terrain situé à seulement quelques dizaines de mètres du rivage. Or, selon une tradition locale, il s'y trouvait anciennement un sanctuaire tamoul, ou kōvila en singhalais, d'où le nom de Kōvilavatta donné à ce lieu. D'anciens vestiges de temple y sont d'ailleurs encore observables au début du $\mathrm{Xx}^{\mathrm{e}}$ siècle, et c'est au milieu de ces derniers qu'une jarre en terre cuite contenant aussi bien la statuette khmère que d'autres objets a été enterrée à faible profondeur ${ }^{27}$.

Grâce à des sources à la fois textuelles et archéologiques, les ruines de Kōvilavatta ont été depuis identifiées comme un sanctuaire dédié à Gaṇeśa, autour duquel existent d'autres temples d'obédience shivaïte. Le fait que la jarre mise au jour dans ce sanctuaire contenait non seulement des figurines de la divinité qui y était honorée, mais aussi des statuettes de Śiva et de Pārvatī ainsi que des linga miniatures, ne semble donc guère étonnant (fig. 8a-b). À côté de cet ensemble religieux tourné vers la mer existe, plus à l'intérieur des terres, au sein même de l'ancienne ville de Dondra, le Khadirālī Vihāra, qui aurait été fondé dès le $\mathrm{VII}^{\mathrm{e}}$ siècle et aurait constitué un très vaste complexe monastique, avec ses lieux saints caractéristiques. Enfin, encore plus au nord et en dehors de l'espace urbain se trouve un sanctuaire lui aussi édifié au VII ${ }^{\mathrm{e}}$ siècle, le célèbre Upulvan Dēvāle. Sa divinité principale, très certainement le dieu des eaux Varuṇa, connaît pendant près d'un millénaire une grande renommée. Celle-ci culmine sans doute $a u \mathrm{XV}^{\mathrm{e}}$ siècle, lorsque arrivent par mer et par terre des pèlerins aussi bien singhalais qu'étrangers. Cette période faste s'achève brutalement à la fin du siècle suivant, lorsque les trois groupes distincts de sanctuaires sont pillés, démolis et incendiés par les Portugais, qui mènent des raids sur les côtes du Sri Lanka.

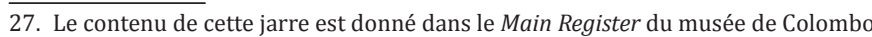
comme suit: une lampe brûle-encens en bronze (15.106.314); une statuette de Pārvatī en bronze ou en cuivre (15.107.314 [fig. 8b]) : une statuette de Śiva et de Pārvatī en stéatite ou en terre cuite (15.112.314); deux linga en stéatite ou en terre cuite (15.113.314 et 15.114.314); trois Ganeśa en stéatite ou en terre cuite $(15.108 .314,15.110 .314$ et 15.111 .314$)$; et un Ganeśa, incomplet, en terre cuite (15.109.314 [fig. 8a]). Seuls cinq objets sont encore conservés dans les réserves du musée (15.105.314, 15.107.314, 15.109.314, 15.112314 et 15.113.314). Les autres pièces ont été envoyées en 1927 au Viṣnu Dēvāle de Dondra, où elles se trouveraient toujours, déposées au sein de la cella du sanctuaire $(15.106 .314,15.108 .314$ $15.110 .314,15.111 .314$ et 15.114.314). Or, cette dernière reste très difficile d'accès, car elle n'est ouverte qu'une fois par an, à l'occasion du Devundara Perahera, festival célébré dans le courant du mois d'août en l'honneur du dieu Vişnu. Il n’a donc pas été possible de mener une étude plus approfondie de l'ensemble des objets du dépôt, ce qui explique les problèmes d'identification de leurs matériaux. Seules deux pièces conservées à Colombo ont été brièvement observées et photographiées, ce qui a permis de remarquer que leur facture était nettement sud-indienne (fig. 8a-b).
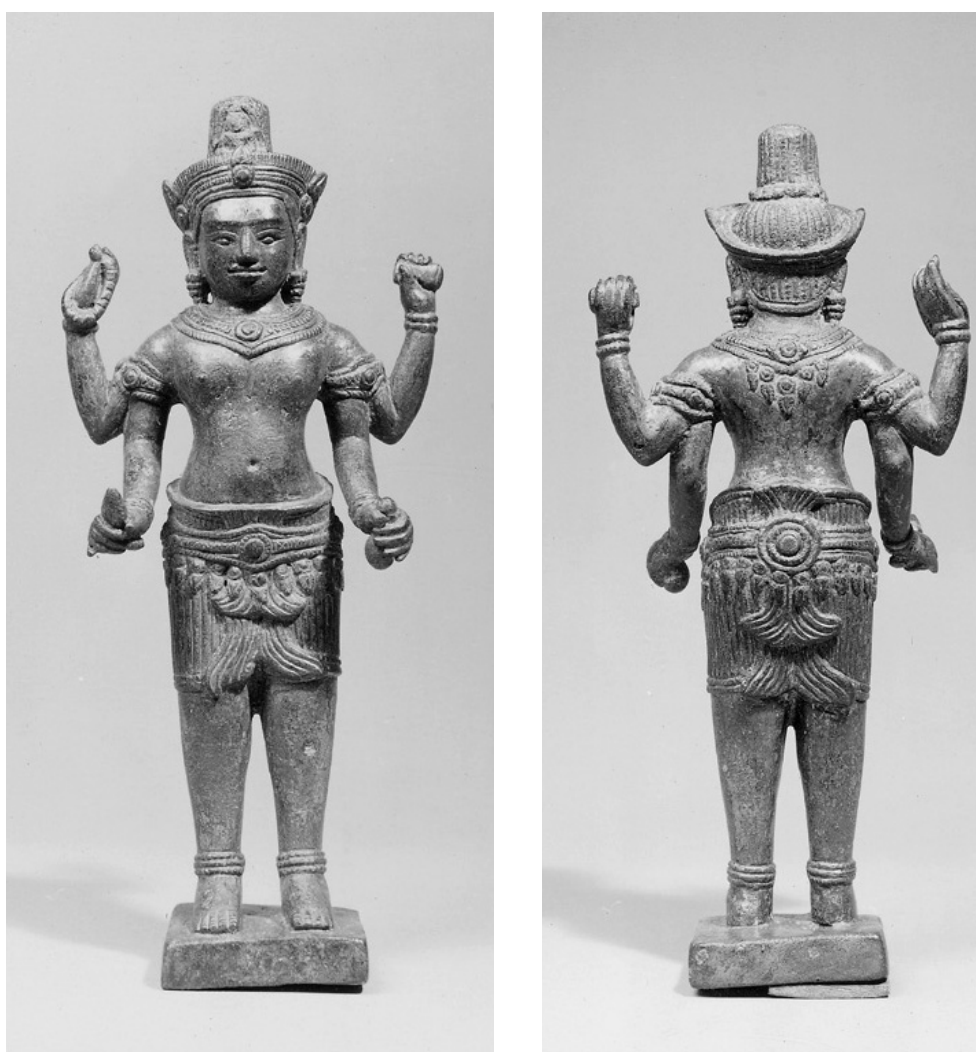

Figures 6a et 6b. - Lokeśvara (face et dos), style du Bayon, fin XII ${ }^{\mathrm{e}}$-début XIII ${ }^{\mathrm{e}}$ siècle, Veal Kralanh, région de Roluos/Angkor, province de Siem Reap (Cambodge). Bronze avec traces de dorure, H. $27 \mathrm{~cm}$. Musée national du Palais, Taipei. Photos École française d'Extrême-Orient, fonds Cambodge, réf. CAM 3251_1 et 3251_2.

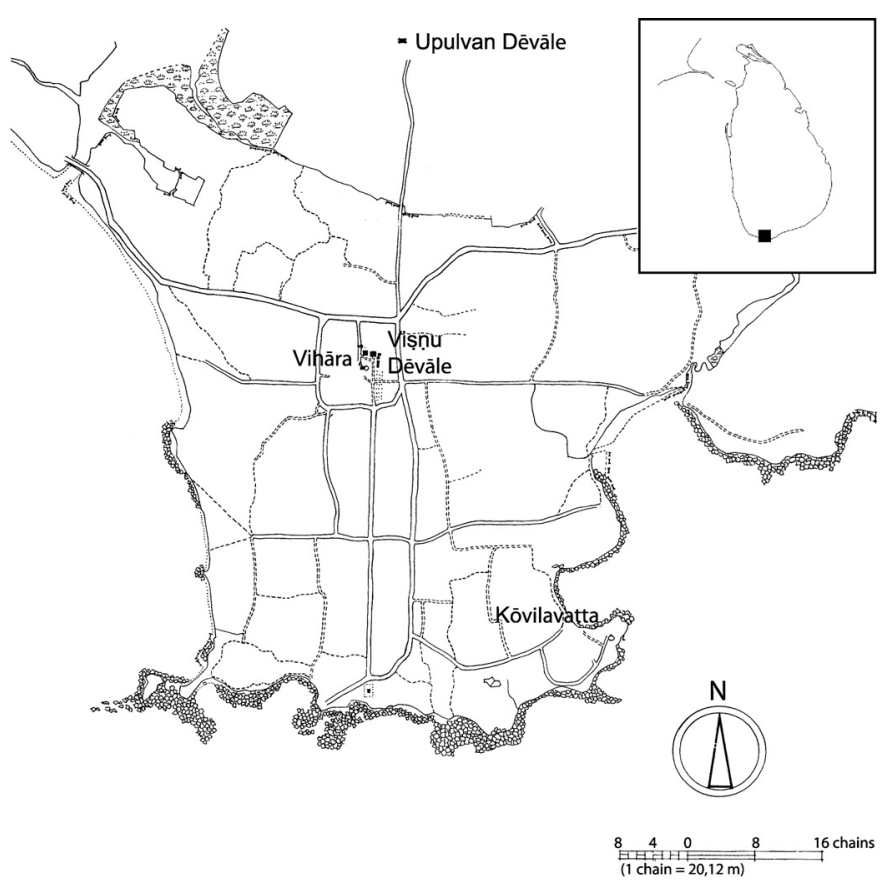

Figure 7. - Plan de Dondra, district de Matara (Sri Lanka).

D'après PARANAVITANA 1953, pl. 16. 


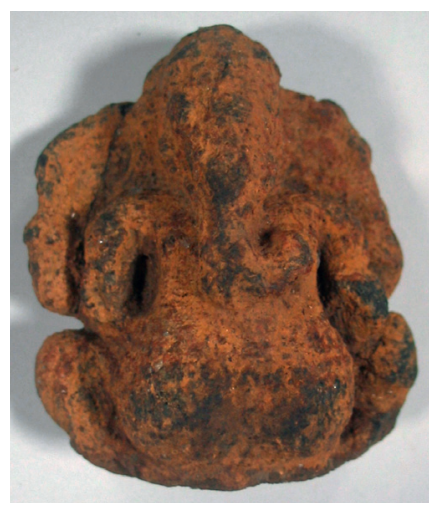

Figure 8a. - Gaṇeśa (incomplet), Kōvilavatta, Dondra, district de Matara (Sri Lanka). Production sudindienne. Terre cuite, H. 7,5 cm. Musé national de Colombo, 15.109.314.

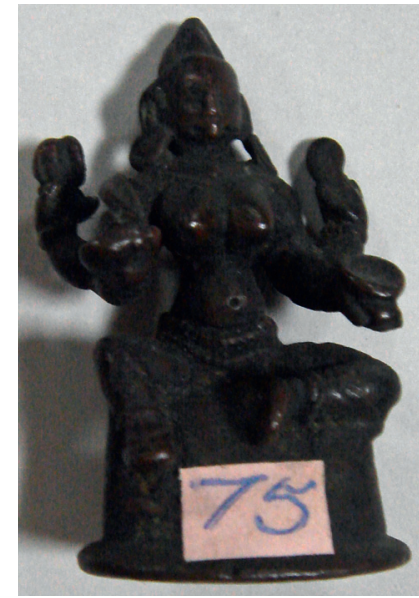

Figure 8b. - Pārvatī, Kōvilavatta, Dondra, district de Matara (Sri Lanka). Production sud-indienne. Bronze ou cuivre, H. 4,5 cm. Musée national de Colombo, 15.107.314.

Plus tard, dans le courant du XVII ${ }^{\mathrm{e}}$ siècle, un nouveau temple dédié à Viṣnu, successeur de l'Upulvan Dēvāle, est édifié à l'emplacement de l'ancien vihāra, qui est lui aussi reconstruit ${ }^{28}$.

Le sac commis par les Portugais n'est pas sans importance, car il fournirait une explication à la présence d'une cache d'objets sur le site de Kōvilavatta. Rappelons à ce propos un autre cas d'enfouissement attesté à Dondra. Une sorte de bol à couvercle en cuivre a en effet été enterré à faible profondeur à proximité de l'ancien Upulvan Dēvāle. Il contenait un trésor de 402 larins portugais en argent et de dix petites pièces en or, d'un type non encore identifié. Un tel trésor est très certainement dû à des habitants cherchant non seulement à fuir la ville au moment du raid portugais, mais aussi à protéger leurs biens ${ }^{29}$. Sans doute en est-il de même pour la jarre mise au jour à Kōvilavatta. Dans ce cas, cependant, en plus d'une valeur intrinsèque, au moins évidente pour la statuette khmère qui a été importée de l'étranger, les images et objets de culte qui se trouvaient à l'intérieur sont aussi porteurs d'une valeur sacrée. La jarre aurait donc été placée dans l'enceinte du sanctuaire dédié à Gaṇeśa, afin de constituer un dépôt sacré, qui aurait ainsi été protégé de la destruction et du pillage.

28. PARANAVitana 1953, p. 11-18. Lors de ces travaux, plusieurs vestiges de l'ancien sanctuaire de Gaṇeśa furent remployés, qu'il s'agisse d'éléments d'architecture ou de statuaire. Selon les Ceylon Administration Reports pour l'année 1915, il se trouve notamment parmi ces remplois un piédroit inscrit daté de 812 A. D. V. Goloubew l'a pris à tort pour un des objets découverts avec le Lokeśvara khmer, ce qui a sans doute contribué à conforter sa première datation de la statuette autour $\mathrm{du}_{\mathrm{IX}}^{\mathrm{e}}$ ou du $\mathrm{x}^{\mathrm{e}}$ siècle (Goloubew 1924, p. 512).

29. Paranavitana 1953, p. 8, pl. 13.

\section{Victor Goloubew et le Roi lépreux : entre légendes et Histoire}

Pour expliquer la découverte d'un Lokeśvara khmer à Dondra, V. Goloubew avance d'abord l'hypothèse d'une offrande faite par un souverain khmer, proche de la royauté singhalaise, à un sanctuaire particulièrement réputé de l'île dédié au bodhisattva $a^{30}$. Il s'emploie toutefois assez rapidement à reformuler cette hypothèse et, dans le cadre de ses recherches menées au Cambodge sur le Roi lépreux, fait de la statuette l'une des pièces à ajouter au dossier de l'historicité de ce personnage légendaire, qu'il finit par reconnaître en la figure de Jayavarman VII ${ }^{31}$.

V. Goloubew croit en particulier, ou tout du moins veut croire, au caractère historique d'un voyage qu'aurait fait ce souverain en Inde et au Sri Lanka pour être guéri de son mal. Du côté du monde khmer, il s'appuie d'abord sur un bas-relief du Bayon, situé sur la face nord de la galerie intérieure du sanctuaire, qui représente la traversée d'un océan par deux personnages principaux (un roi et un ascète?), et leur arrivée aux pieds d'une montagne défendue par des nāga et des animaux sauvages (fig. 9a-b). Il voit en effet dans cette scène une illustration du voyage de Jayavarman VII et fonde son interprétation sur le Khambhōjarājacaritram, un manuscrit en langue telugu racontant justement l'histoire d'un roi du Cambodge (Khambhōja), qui aurait fait un pèlerinage en Inde et aurait été miraculeusement guéri de la lèpre sur le mont Meru ${ }^{32}$. Du côté du monde indien, V. Goloubew se rappelle en outre la visite d'un site archéologique du sud du Sri Lanka, Weligama, situé à environ vingt kilomètres à l'ouest de Dondra, toujours dans le district de Matara. Il y avait notamment observé une statue monumentale du bodhisattva Avalokiteśvara sculptée sur un rocher, à laquelle une tradition locale donne le nom de Kușțarāja ou « Roi lépreux » ${ }^{33}$ (fig. 10). Quelques

\section{Goloubew 1924, p. 512.}

31. Si V. Goloubew n'a jamais consacré un article, ni même une note, au Roi lépreux, alors que ce sujet a longtemps constitué le leitmotiv de ses recherches, il reste possible de saisir l'avancée de ses questionnements en consultant plusieurs de ses articles, ou encore les travaux de certains de ses collègues qui y font référence. Dans un article déjà cité, D. Chandler fournit une liste quasi exhaustive de ces publications ainsi qu'un résumé des recherches de V. Goloubew sur la question (CHANDLER 1979, p. 58-59). Pour être complet, il faut encore ajouter plusieurs documents conservés dans les archives personnelles de V. Goloubew, aujourd'hui déposées à la bibliothèque de l'EFEO à Paris («Dossier Victor Goloubew » : carton D.1, dossier R.5, "Documents relatifs au Roi lépreux»; carton D.1, dossier R.63, "Correspondance 1920-1945»).

32. MALLERET 1967, p. 353; $c f$ aussi CoEDÈs 1937 p. 27. Ce manuscrit en langue telugu, et non en langue tamoule comme V. Goloubew l'indique parfois, lui a été signalé par Sylvain Lévi, qui l'avait identifié quelques années plus tôt dans la Government Oriental Manuscripts Library de l'université de Madras. Il y est toujours conservé, mais figure dans l'inventaire des manuscrits telugu sous le titre de Kāmbhōjarāju kathalu (D.3157; Kuppuswami SASTRI 1932, nº 510). Le titre qui est écrit sur le manuscrit correspond en revanche à celui donné par V. Goloubew: Kaumbhoja Raja Scharitra (sic). Nous savons par ailleurs que le savant a utilisé une traduction anglaise de ce manuscrit, mais il n'a pas été possible de la retrouver au sein de ses archives personnelles. Parmi les documents disparus, il est néanmoins fait mention d'« un document tout en langue anglaise à traduire de G. V. à l'EFEO », qui pourrait être la traduction en question.

33. Cette statue, dont l'iconographie et la datation ont longtemps posé problème, est désormais clairement identifiée comme une forme d'Avalokiteśvara et attribuée 


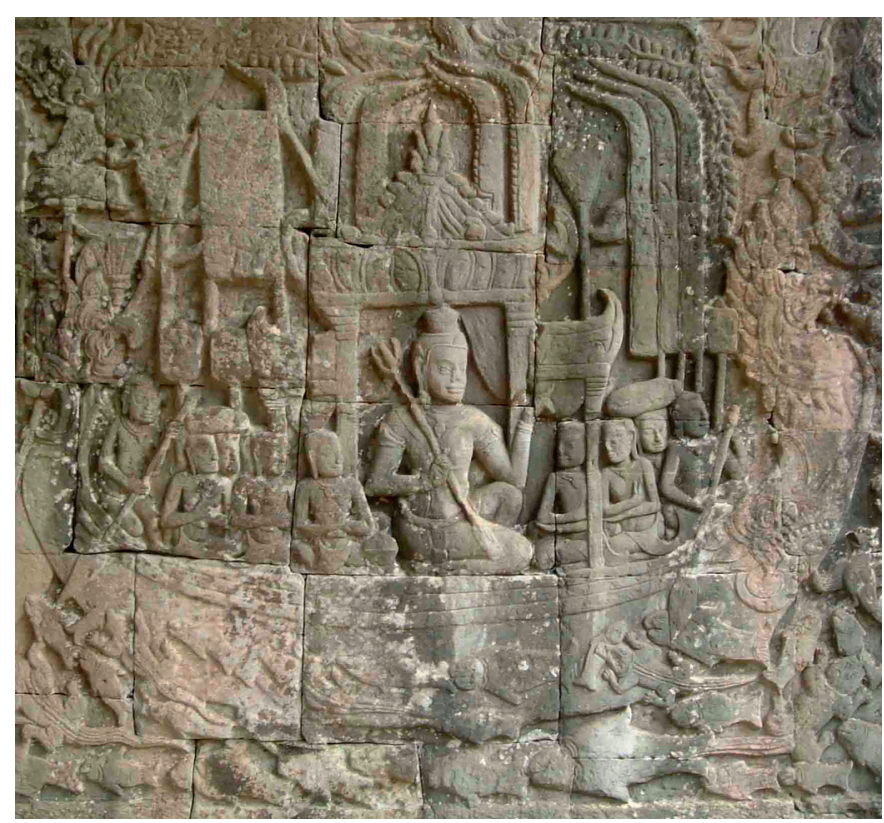

De gauche à droite :

Figure $9 a$. - Bas-relief représentant la traversée d'un océan par un roi (?), XIII ${ }^{\mathrm{e}}$ siècle (?), Bayon (galerie intérieure, face nord, côté ouest), Angkor Thom, province de Siem Reap (Cambodge)

Figure 9b. - Bas-relief représentant une montagne défendue par des nāga et des animaux sauvages (mont Meru ?), XIII ${ }^{\mathrm{e}}$ siècle (?), Bayon (galerie intérieure, face nord, côté ouest), Angkor Thom, province de Siem Reap (Cambodge)

années plus tard, il apprend surtout de son maître Sylvain Lévi que cette dernière aurait été sculptée, en guise d'ex-voto, par un souverain étranger, lui aussi miraculeusement guéri de la lèpre ${ }^{34}$. Le pèlerinage en Inde et au Sri Lanka du Roi lépreux «historique», Jayavarman VII, serait ainsi attesté par une tradition tant écrite qu'orale, à laquelle pourrait en plus s'ajouter un témoignage archéologique plus ou moins contemporain : le Lokeśvara khmer de Dondra, découvert non loin de Weligama.

Si cette accumulation de faits et légendes est séduisante, plusieurs arguments peuvent être avancés pour aller à l'encontre de la reconstruction historique proposée par V. Goloubew. Tout d'abord, il ne faut pas oublier que les bas-reliefs de la galerie intérieure du Bayon ne cessent de poser des problèmes de datation et d'identification. Un consensus tend néanmoins à placer la réalisation de la majeure partie de ces bas-reliefs, dont ceux de la face nord, après la mort de Jayavarman VII ${ }^{35}$. Il est donc peu probable que ce dernier soit représenté sur l'un des bas-reliefs précédemment décrits. Par ailleurs, si le souverain a effectivement réalisé ce pèlerinage en Inde et au Sri Lanka et bénéficié

à la période tardive d'Anurādhapura, soit au IX $\mathrm{x}^{\mathrm{e}}$ ou au X $\mathrm{x}^{\mathrm{e}}$ siècle (Von SCHROEDER 1990 p. 221, 294-295, fig. 82b).

34. GoloubEw 1935, p. 566. Cette version diffère sensiblement de celle entendue par V. Goloubew lorsqu'il se rend sur le site de Weligama: selon cette autre tradition locale, le Roi lépreux (Kușțarāja), guéri de son mal, aurait élevé sur le site un sanctuaire en mémoire de sa guérison (FINOT et Goloubew 1930, p. 642). Une autre version est encore rapportée par Senarat Paranavitana et complète de manière intéressante celle de S. Lévi : c'est, cette fois, l'image sculptée sur le rocher qui représenterait un souverain étranger. Ce dernier, souffrant de la lèpre, avait quitté son pays d'origine, avant de débarquer à Weligama et d'être guéri sur place par un médecin (PARANaVitana 1928, p. 49).

35. Roche-Provost 2010, p. 88-113.

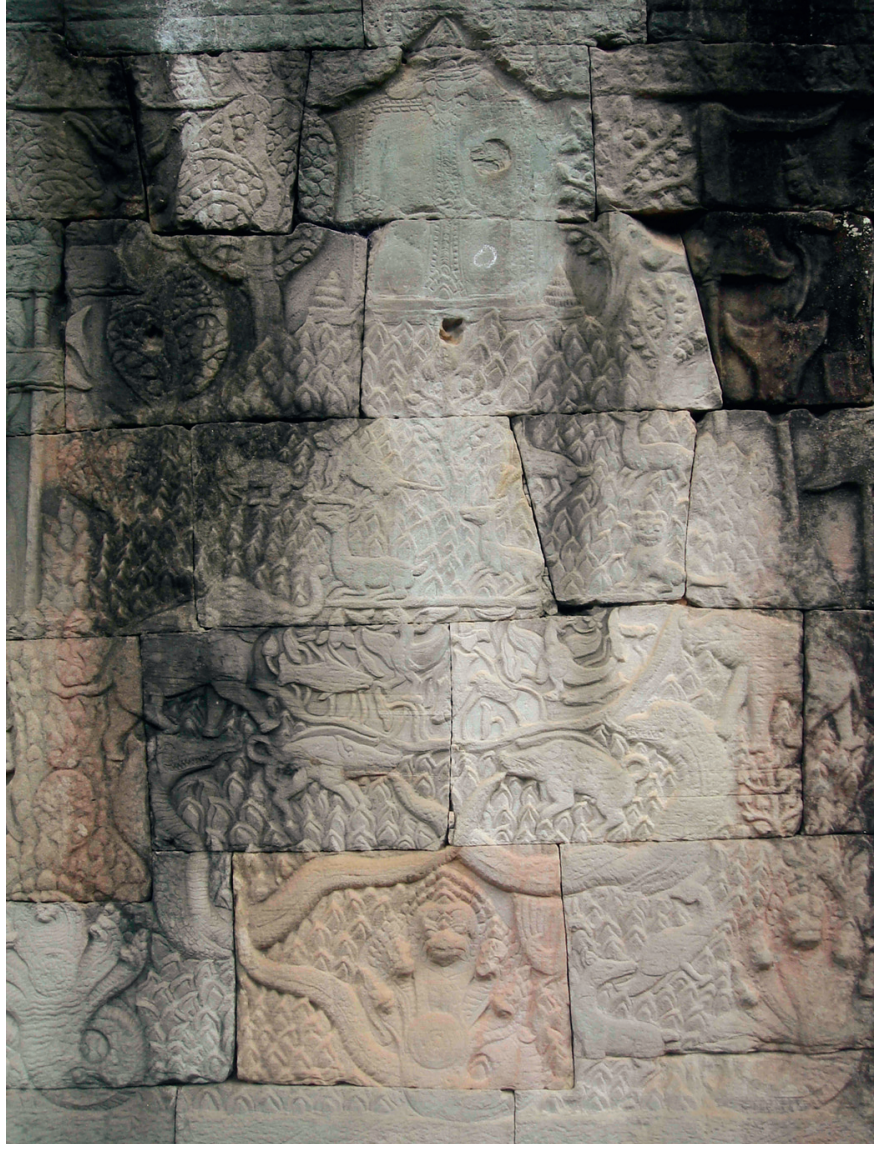

d'une guérison miraculeuse, il en aurait très certainement laissé un récit dans l'une des nombreuses inscriptions de son règne ${ }^{36}$. Enfin, le manuscrit en langue telugu du Khambhōjarājacaritram sur lequel s'appuie V. Goloubew est fort problématique. Trois autres manuscrits répondant au même titre sont connus en Inde et tous relatent les histoires d'un roi de Khambhōja. Il s'agit là d'un topos de la littérature et du folklore telugu. Cependant, ce terme de Khambhōja renvoie toujours à une région située dans le nord-ouest de l'Inde, et non au Cambodge ${ }^{37}$. Une nouvelle traduction de ce manuscrit permettrait sans doute de savoir si la tradition écrite qu'il représente a servi ou non de source d'inspiration, plus ou moins lointaine, aux sculpteurs khmers qui ont travaillé au Bayon ${ }^{38}$.

36. Ces deux derniers arguments ont déjà été avancés par D. Chandler, mais surtout pour invalider l'identification que fait V. Goloubew du Roi lépreux avec Jayavarman VII (CHANDleR 1979, p. 59-61). L'auteur ne récuse pas en effet l'idée du pèlerinage d'un souverain khmer atteint de la lèpre en Inde et au Sri Lanka, mais préfère voir dans ce dernier le successeur de Jayavarman VII, Indravarman II (r. ca. 1220-1270). Il serait ainsi à identifier sur les bas-reliefs de la galerie intérieure du Bayon et, en particulier, sur ceux du pavillon d'entrée oriental que V. Goloubew a, le premier, proposé d'associer avec le cycle légendaire du Roi lépreux (Roche-Provost 2010, p. 101-102, 111-112). Toujours est-il que la grande composition de la face nord, qui est censée faire suite à ces premières scènes et représenter le pèlerinage du Roi lépreux, montre deux personnages principaux nettement distincts de celui représenté sur les basreliefs de la face est, aussi bien par leur apparence physique que par leur vêtement (Roche-Provost 2010, p. 95). Elle figurerait donc un épisode sans rapport aucun avec la légende du Roi lépreux, ce qui éliminerait par là la seule preuve disponible côté khmer du voyage d'Indravarman II en Inde et au Sri Lanka.

37. Communication personnelle de Madabushi Sampath Kumar, professeur de telugu à l'université de Madras, juin 2008.

38. Dans ce but, une copie numérique du manuscrit a été réalisée lors de notre dernier passage à Chennai. Il est désormais à espérer que des spécialistes de telugu puissent s'employer à identifier les différentes scènes contenues dans le récit. 
il s'installe à Pagan, afin de propager l'enseignement qu'il a reçu ${ }^{42}$. L'identité de Tāmalinda a toutefois récemment fait l'objet d'une nouvelle interprétation, selon laquelle ce moine serait originaire, non pas du Cambodge, mais de Basse Birmanie, comme d'ailleurs les quatre anciens compagnons qui ont fait le voyage avec lui jusqu'à Pagan. Les chroniqueurs birmans auraient volontairement faussé son origine ainsi que celle de ses compagnons, afin de représenter à travers eux une sorte de carte religieuse du bouddhisme Theravāda à la fin du XII ${ }^{\mathrm{e}}$ siècle : de fait, celle-ci comprend le Cambodge avec Tāmalinda, le Sri Lanka avec Rāhula, l'Inde du Sud avec Ānanda, le Bengale avec Sivvalī et, bien évidemment, la Birmanie avec Chapața, dont la prééminence est implicitement reconnue $^{43}$. Progressivement inséré au sein de ce réseau bouddhique theravādin au cours de la seconde moitié du XII ${ }^{\mathrm{e}}$ siècle, le pays khmer aurait été mis en relation avec le Sri Lanka, et un certain nombre de pèlerins, autres que le célèbre mais controversé Tāmalinda, auraient fait le voyage vers l'île. Depuis la réforme du bouddhisme singhalais par le souverain Parākramabāhu I ${ }^{\text {er }}$ et son unification sous la seule foi theravādin, elle ne cesse en effet d'attirer des moines étrangers, désireux de venir étudier la nouvelle orthodoxie singhalaise ou de recevoir l'ordination ${ }^{44}$.

De telles interactions religieuses, si elles ont bien existé, auraient aidé à faire connaître jusqu'en pays khmer la renommée du sanctuaire mahāyānique de Weligama et de son bodhisattva guérisseur ${ }^{45}$. À moins que les premiers contacts politiques établis entre les royaumes khmer et singhalais y aient eux aussi contribué. L'intérêt des bouddhistes khmers pour ce sanctuaire aurait en tout cas été d'autant plus grand que, dans cette même seconde moitié du XII ${ }^{\mathrm{e}}$ siècle, le Cambodge voit l'essor d'un nouveau culte dédié à Lokeśvara. La divinité y est même spécifiquement vénérée en tant que bodhisattva guérisseur à partir de la fin du siècle ${ }^{46}$. En suivant toujours l'hypothèse d'un pèlerin bouddhiste khmer porteur de l'idole de Lokeśvara, nous disposons ainsi d'un possible terminus post quem pour son voyage du Cambodge vers le Sri Lanka, qui ne s'oppose pas à la date de production attribuée à la statuette ${ }^{47}$.

42. SIRISENA 1978, p. 104-105; cf. aussi ILANGASINHA 1998

43. FRASCH 2000, p. 65.

44. Chutiwongs 2002, p. 55-56, 58-59.

45. N'oublions pas en effet qu'au Sri Lanka, malgré l'affirmation du bouddhisme Theravāda, les cultes mahāyāniques, et notamment celui du bodhisattva Avalokiteśvara continuent d'être pratiqués. Ce n'est qu'à partir du XIV ${ }^{\mathrm{e}}$ siècle que la divinité est progressivement assimilée. Dans plusieurs sanctuaires theravādin datant de cette époque, des images d'Avalokiteśvara sont alors installées à côté de celles du Buddha ou de Maitreya. C'est peut-être dans ce contexte religieux que la statuette khmère de Lokeśvara aurait été remployée à Dondra, au sein d'un des lieux saints du Khadirālī Vihāra, mais aussi reprise afin de faire disparaître l'image miniature du Buddha Amitābha figurant à l'avant de sa coiffure.

46. Chutiwongs 2002, p. 220-221

47. Si l'on décide néanmoins de laisser de côté cette hypothèse, à nos yeux la plus vraisemblable, d'autres explications peuvent être avancées. Passée de main en main, la statuette khmère de Lokeśvara aurait été apportée plusieurs siècles plus tard sur l'île, par un moine theravādin du Siam ou encore de Birmanie, des relations étroites existant alors entre les communautés bouddhiques de ces différentes régions. Mais sa présence au Sri Lanka pourrait aussi ne pas forcément répondre à un acte reli-

\section{Réseaux d'échanges régionaux et «bronzes voyageurs »}

Si l'origine et la destination de ce voyage sont connues, plusieurs parcours restent enfin à envisager, en prenant en compte les principaux réseaux d'échanges en place en Asie du Sud et du Sud-Est au cours de la période étudiée.

Un intermédiaire birman peut d'abord être proposé, ce qui implique une route à la fois terrestre et maritime intégrant le vaste réseau bouddhique theravādin précédemment évoqué. C'est cette route que tentent notamment de suivre les envoyés du souverain singhalais Parākramabāhu I ${ }^{\mathrm{er}}$. Un autre relais possible est la péninsule Malaise et, en particulier, la cité-État du Tāmbralinga, qui, outre un port-entrepôt, aurait constitué un important centre d'études bouddhiques entre 1130 et 1176 . Ces dates sont fournies par les chroniques de Nakhon Si Thammarat et correspondent à la période présumée de domination singhalaise sur la ville, avant qu'elle ne tombe sous contrôle birman ${ }^{48}$. Si des relations étroites sont attestées avec le Sri Lanka - et la Birmanie -, elles existent aussi côté khmer, ce dont témoignent plusieurs siècles d'interactions politiques et commerciales entre les deux rives du golfe de Thaïlande.

Une série d'objets de facture khmère, ou d'inspiration khmère, ont d'ailleurs été découverts sur la côte est de la péninsule Malaise. Ils ont sans doute été laissés par des marchands, mais peut-être aussi par quelques pèlerins venus honorer une ou plusieurs divinités locales. Parmi ce mobilier archéologique, il faut retenir plusieurs statuettes en bronze, aussi bien bouddhiques que brahmaniques, qu'il est permis de dater entre la seconde moitié du $\mathrm{X}^{\mathrm{e}}$ et le début du XIII ${ }^{\mathrm{e}}$ siècle $^{49}$. Tout en renseignant sur les réseaux et les routes actifs dans la région, ces images de culte essentiellement mobiles ont aussi l'intérêt de montrer que la statuette du musée de Colombo n'est pas le seul exemple connu pour le monde khmer de «bronze voyageur».

\section{Brice Vincent, Center for Khmer Studies, Siem Reap bricevincent@yahoo.fr}

gieux. Au vu de son caractère isolé, elle aurait en effet été ramenée au simple titre de " curiosité » par un marchand, sud-est asiatique ou autre.

48. JACQ-HERGOUALC'H 2002, p. 399-401.

49. JACQ-HERGOUALC'H 2002, p. 380-381, 402-404, fig. 181-182, 196-199. 


\section{Bibliographie}

\section{Archives}

Ceylon Administration Reports, 1916 : «The Colombo Museum. Report of the Director for 1915 », Ceylon Administration Reports, 1915, Colombo, H. C. Cottle (Government Printer), D 4.

Main Register et Section Registers: Colombo, Colombo National Museum.

« Dossier Victor Goloubew »: Paris, bibliothèque de l'EFEO (carton D.1, dossier R.5, «Documents relatifs au Roi lépreux » ; carton D.1, dossier R.63, «Correspondance 1920-1945»).

\section{Sources anciennes}

GEIGER Wilhelm (éd.), 1925-1927 : Cülavamsa, being the more recent part of the Mahāvamisa, 2 vol., Londres, Oxford University Press (Pali Text Society).

Kaumbhoja Raja Scharitra: Various Histories on the Hindou Religion relat[ed] to Kaumbhoja Raja (a Prince of Kaumbhoja Dus[_n]) By Datutraya Moony [Kāmbhōjarāju kathalu], Chennai, université de Madras, Government Oriental Manuscripts Library B.712 [inventaire général] ; D.3157 [inventaire des manuscrits en langue telugu]), $53 \mathrm{p}$.

Kuppuswami SASTRI S., 1932 : An alphabetical index of Telugu manuscripts in the Government Oriental Manuscripts Library, Madras, Madras, Government Press.

Pe Maung Tin et Luce Gordon H. (trad.), 1960 : The Glass Palace Chronicle of the Kings of Burma, Rangoon, Rangoon University Press.

\section{Travaux modernes}

BAPTISTE Pierre et ZÉPHIR Thierry, 2008: L'art khmer dans les collections du musée Guimet, Paris, Réunion des musées nationaux.

BEFEO, 1923: Chronique, "France», BEFEO, 23, p. 559-569.

BEFEO, 1925 : Chronique, « Cambodge », BEFEO, 25 (1-2), p. 276-278.

BRIGGS Lawrence P., 1951: The Ancient Khmer Empire, Philadelphia, American Philosophical Society.

CHANDLER David P., 1979: «Folk memories of the decline of Angkor in nineteenth century Cambodia: the legend of the Leper King », Journal of the Siam Society, 67 (1), p. 54-62.
Chutiwongs Nandana, 2002: The Iconography of Avalokiteśvara in Mainland Southeast Asia, New Delhi, Aryan Books International.

CoEDÈs George, 1923 : Bronzes khmèrs. Étude basée sur des documents recueillis par M. P. Lefèvre-Pontalis dans les collections publiques et privées de Bangkok et sur les pièces conservées au Palais Royal de Phnom Penh, au Musée du Cambodge et au Musée de l'EFEO, Paris-Bruxelles, Gérard Van Oest (Ars Asiatica).

- 1937: «Le Mystère du Bayon d'Angkor Thom», CEFEO, 10, p. 25-30.

- 1964a: Inscriptions du Cambodge, vol. 7, Paris, EFEO.

- 1964b : Les États hindouisés d'Indochine et d'Indonésie, Paris, De Boccard.

FINOT Louis et GoloubEW Victor, 1930 : «Rapport sur une mission archéologique à Ceylan », BEFEO, 30 (3-4), p. 627-643.

FRASCH Tilman, 2000 : «Die Welt des Buddhismus im Jahre 1000 », in KuLKe Hermann (éd.), Asien im Jahre 1000, Hamburg, LIT Verlag, p. 56-72.

Goloubew Victor, 1924 : « Mélanges sur le Cambodge ancien, II. Une idole khmère de Lokeçvara au musée de Colombo », BEFEO, 24 (3-4), p. 510-512.

-1930 : « The art of India and Indo-China with special reference to Ceylon », Journal of the Royal Asiatic Society (Ceylon Branch), 31 (83), p. 455-464.

- 1935 : «Sylvain Lévi et l'Indochine », BEFEO, 35 , p. 551-567.

GRosLIER Bernard Philippe, 1995 : «Introduction à la céramique angkorienne (fin $\mathrm{IX}^{\mathrm{e}}$-début $\mathrm{XV}^{\mathrm{e}}$ siècle) 》, Péninsule, 31 (2), p. 5-60.

Ho Christine, Lin Boting, Zhu Linze et al. (éd.), 2004 : The Casting of Religion: A special exhibition of Mr Peng Kai-dong's donation, Taipei, National Palace Museum.

ILANGASINHA Mangala, 1998: «Some aspects of relations between Sri Lanka and Cambodia », in SoRN Samnang (éd.), Khmer studies: Knowledge of the past and its contributions to the rehabilitation and reconstruction of Cambodia, Proceedings of International Conference on Khmer Studies, Phnom Penh, 26-30 August 1996, vol. I, Phnom Penh, Ministry of Education, Youth and Sports/Royal University of Phnom Penh, p. 186-198.

JacQ-Hergoualc' Hichel, 2002 : The Malay Peninsula: Crossroads of the Maritime Silk Road (100 BC-1300 $A D)$, Leiden, E. J. Brill.
JACQuEs Claude, 1967: "Note sur une stèle d'Avalokiteśvara à Gayā », Arts Asiatiques, 16, p. 119-124.

LE BonHEuR Albert, 1972 : «Un bronze d'époque préangkorienne représentant Maitreya », Arts Asiatiques, 25, p. 129-154.

MALLERET Louis, 1967 : « Le vingtième anniversaire de la mort de V. Goloubew (1878-1945) », BEFEO, 53 (2), p. 331-373.

PARANAVITANA Senarat, 1928 : « Mahāyānism in Ceylon », Ceylon Journal of Science (Section G), 2 (1), p. 35-71.

- 1953: The Shrine of Upulvan at Devundara, Colombo Archaeological Department, Ceylon Goverment.

Roche-Provost Ludivine, 2010 : « Les derniers siècles del'époque angkorienne au Cambodge (env. 1220 env. 1500) », 2 vol., Thèse de doctorat, Paris, université Sorbonne nouvelle-Paris 3.

SiRISENA W. M. 1978 : Sri Lanka and South-East Asia: Political, Religious and Cultural Relations from A.D. c. 1000 to c. 1500 , Leiden, E. J. Brill.

VINCENT Brice, 2012 : «Samrit. Étude de la métallurgie du bronze dans le Cambodge angkorien (fin du XI ${ }^{\mathrm{e}-}$ début du XIII ${ }^{\mathrm{e}}$ siècle) », 3 vol., Thèse de doctorat, Paris, université Sorbonne nouvelle-Paris 3.

SchroEdER Ulrich von, 1990 : Buddhist Sculptures of Sri Lanka, Hong Kong, Visual Dharma Publications.

WoodWARD Hiram W., Jr., 1980 : « Some Buddha images and the cultural developments of the late Angkorian period », Artibus Asiae, 42 (2-3), p. 155-174.

- 1981: «Tantric Buddhism at Angkor Thom», Ars Orientalis, 12, p. 57-67.

ZÉPHIR Thierry, 2012: « Notes à propos d'un mandala d'Hevajra conservé au Musée national de PhnomPenh", in LefÈvre Vincent (éd.), Orientalismes. De l'archéologie au musée. Mélanges offerts à JeanFrançois Jarrige, Turnhout, Brepols, p. 405-425. 